\title{
Research on InGaN/GaN Solar Cells Using Thoma Swan CCS-MOCVD System
}

\author{
Yu Tongwei \\ Institute of Electric Power Science \\ State Grid Liaoning Electric Power Company Limited \\ Shenyang, China \\ yu_twei@hotmail.com \\ Sui Yuqiu \\ Institute of Electric Power Science \\ State Grid Liaoning Electric Power Company Limited \\ Shenyang, China \\ Suiyuqiu_sgcc@163.com
}

\author{
Huang Xu \\ Institute of Electric Power Science \\ State Grid Liaoning Electric Power Company Limited \\ Shenyang, China \\ e-mail: huangxu_sy@126.com \\ Wang Chenggang \\ State Grid Liaoning Electric Power Company Limited \\ Shenyang, China \\ e-mail: wangchenggang@sgcc.com.cn
}

\begin{abstract}
Two InGaN/GaN MQWs samples were grown with two different showerhead gap position 13 and $25 \mathrm{~mm}$, using Thoma Swan CCS-MOCVD system, in order to study the effect of showerhead gap position on the growth of InGaN/GaN MQWs. Through the measurement results, we find that the surface morphology, interface quality, thickness of $Q B$ and $Q W$, In composition and $P L$ spectrum of samples are all change significantly. This can attributes to the change of temperature field, flow field and concentration field in the reactor due to the different showerhead gap position. Moreover, the showerhead gap position also affect the pre-reaction in the reactor, which result in converting the thickness of $Q B, Q W$ and In composition. Consequently, it make the PL spectrum different.
\end{abstract}

Keywords-Solar cell; $M Q W$; gap position; MOCVD; $P L$

\section{INTRODUCTION}

The III-Nitride semiconductor $\operatorname{In}_{\mathrm{x}} \mathrm{Ga}_{1-\mathrm{x}} \mathrm{N}$ has the unique advantage of the widest adjustment of direct bandgaps by In concentration from the infrared ( $\mathrm{InN}$ at $0.65 \mathrm{eV})$ to the ultraviolet (UV) $(\mathrm{GaN}$ at $3.42 \mathrm{eV})$ region [1]. Compared with $\mathrm{Si}, \mathrm{GaAs}$, and other systems, $\mathrm{In}_{\mathrm{x}} \mathrm{Ga}_{1-\mathrm{x}} \mathrm{N}$ is the only semiconductor system that can provide a perfect match to the solar spectrum about $96 \%$, which brings an opportunity for high-efficiency photovoltaic applications. Otherwise, InGaN-based alloys also have the favorable physical properties of high absorption coefficient $\left(\sim 10^{-5} \mathrm{~cm}^{-1}\right)$, high radiation resistance, high drift velocity, and high carrier mobility. The theoretical power conversion efficiency of a multijunction solar cell based on $\mathrm{InGaN}$ is expected to be more than $72 \%$ [2]. Now, many methods such as p-i-n, multijunction tandem, InGaN/GaN multi-quantum well (MQW) are proposed, and MQW is thought to achieve high conversion efficiency.

The metal organic chemical vapor deposition
(MOCVD) method is the most common method for the growth of InGaN/GaN MQW [3-5]. In MOCVD reaction chamber, the height of showerhead has a great influence on the growth rate, utilization and uniformity of metal organic source, its adjustment has been an interesting issue [6]. As early as 1998, Mihopoulos had simulated the gap of close coupling showerhead (base and the showerhead from 10 to $20 \mathrm{~mm}$ ), the results show that the different gap of showerhead brings the different flow rate of reaction gas and mixed degree [7]. Then Aixtron introduced a reaction chamber of Crius (30 machine) system, in which the gap can be lifted, the simulation result shows that the best gap for GaN growth is $11 \mathrm{~mm}$, while the suitable height for InGaN MQW is $13 \mathrm{~mm}$, in this gap, the uniformity of MQW has been significant improved [8]. Zuo has put forward a new type of showerhead of MOCVD reactor, the simulated results confirmed that the showerhead gap significantly influent on the flow field, temperature field and the distribution of gas concentration [9-10].

The recent researches of showerhead gap adjustment mostly focus on the numerical simulation of flow field in the special reaction chamber structure; the related experiment report is rare. There are many influencing factors in the real epitaxial growth, especially for MQW which is sensitive to the growth condition and its influence factors is more complex. Therefore, in this letter, we prepared two InGaN/GaN samples with different showerhead gap in MOCVD, and to study the influence on InGaN/GaN MQW about gap adjustment

\section{METHOD}

Samples in this study were grown on c-plate (0001) sapphire by MOCVD in a Thomas Swan $3 \times 2$ closecoupled showerhead reactor. Hydrogen $\left(\mathrm{H}_{2}\right)$ and nitrogen $\left(\mathrm{N}_{2}\right)$ were used as carrier gas. The trimethylgallium 
(TMGa), triethylgallium (TEGa), trimethylindium (TMIn) and ammonia $\left(\mathrm{NH}_{3}\right)$ were used as $\mathrm{Ga}$, In and $\mathrm{N}$ source. Silicane $\left(\mathrm{SiH}_{4}\right)$ was used as n-type dopant source. The MQW structure consisted of a $20 \mathrm{~nm}$ thick low temperature $\left(530^{\circ} \mathrm{C}\right) \mathrm{GaN}$ buffer, a $1 \mu \mathrm{m}$ undoped-GaN and $3 \mu \mathrm{m}$ Si-doped n-type $\mathrm{GaN}$ (the carrier concentration was $1 \times 10^{19} \mathrm{~cm}^{-3}$ ), 6 pairs of InGaN/GaN MQW, a $20 \mathrm{~nm} \mathrm{GaN}$ cap layer to protect MQW, the growth time of quantum well and quantum barrier is $92 \mathrm{~s}$ and 200s respectively, and the showerhead gap position is 13 and $25 \mathrm{~mm}$. The structure is schematically shown in Figure 1.

\begin{tabular}{|c|}
\hline GaN \\
\hline G'MOWs \\
\hline n-GaN \\
\hline u-GaN \\
\hline GaN buffer \\
\hline Sapphire \\
\hline
\end{tabular}

Figure 1. The schematic of sample structure

\section{RESULTS AND DISCUSSION}

A. In situ monitor
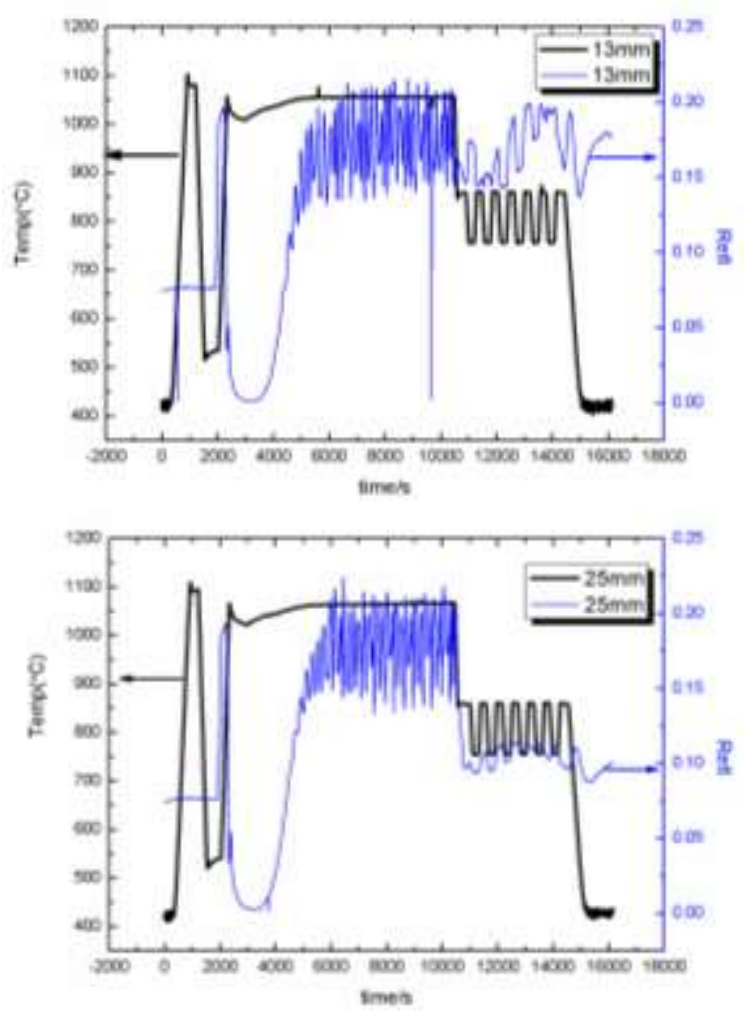

Figure 2. The growth temperature and reflectivity curve of samples

Figure 2 exhibits the growth temperature and reflectivity curve of samples with different gap position. Now, we focus on the step of gap adjustment. When the gap position altered, the reflectivity appears different, and the reflectivity decreases dramatically of $25 \mathrm{~mm}$. Compared with quantum well overall growth stage, as increasing the gap position, the reflectance curve shift down, and the oscillation amplitude curves also showed a trend of decrease. There are many reasons to cause the results, for the growth conditions of MQW surface may be changed, deteriorating the surface quality, thus brings a reflectivity change. The decreasing interference light intensity may be another reason to lead to a lower reflectivity. In addition, during the gap adjustment, the reaction chamber undergo a cooling process, the temperature changes can also cause a certain degree of influence on the reflectivity.
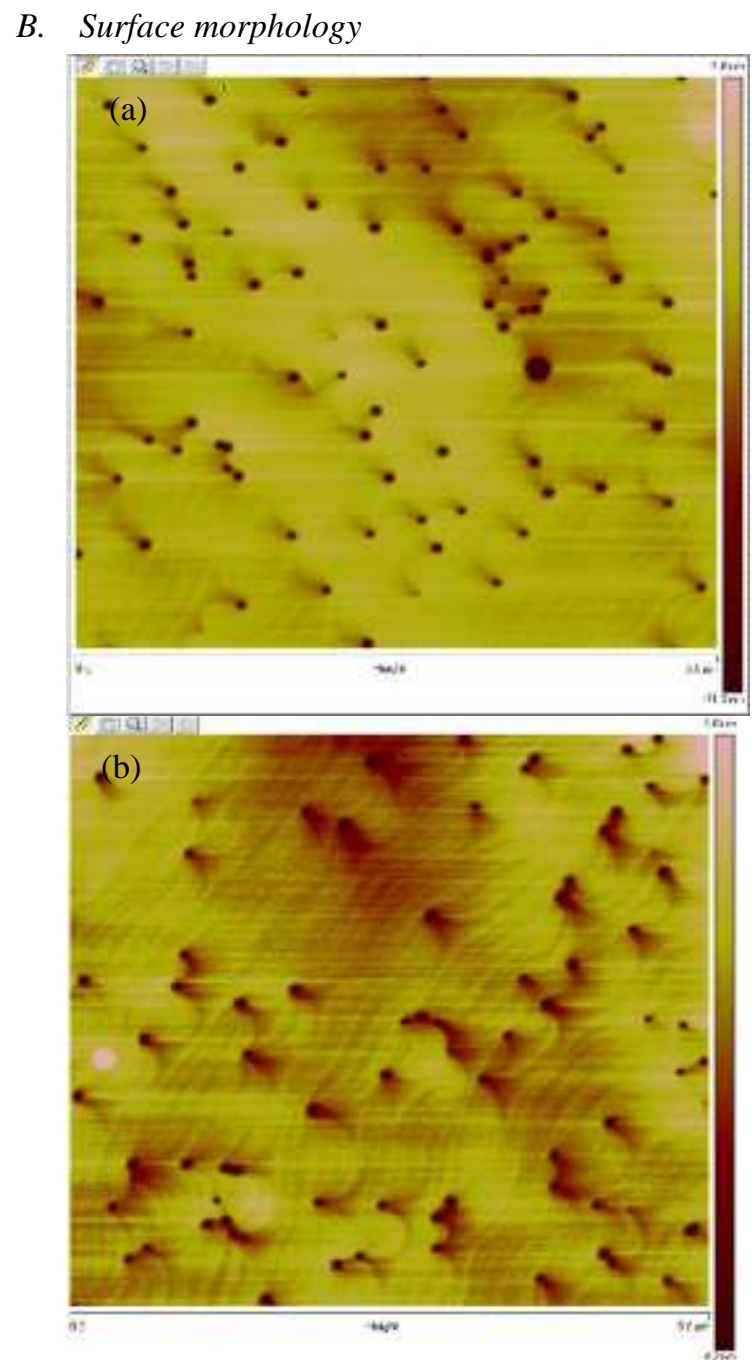

Figure 3. AFM images of the surface morphologies of the samples ((a) $13 \mathrm{~mm}$ (b) $25 \mathrm{~mm}$ )

Figure 3 shows the AFM images of the surface morphologies of two samples, the scanning area is $5 \mu \mathrm{m} \times 5 \mu \mathrm{m}$. With the increase of gap position, the step-flow mode appears clearer, and the surface becomes smoother. The $\mathrm{V}$ defects are observed on both two samples [11-12], these defects exhibit a hexagonal shape, which are thought to be origin from threading dislocation, or can be derived from stacking fault boundary of stacking mismatch [13]. With the increase of gap position, the density of $\mathrm{V}$ defects increases, but the RMS dropped to $0.233 \mathrm{~nm}$ from 0.315 nm. The above phenomenon shows that the gap position has a great influence on the surface roughness and the defect density. 


\section{Crystal charactor}

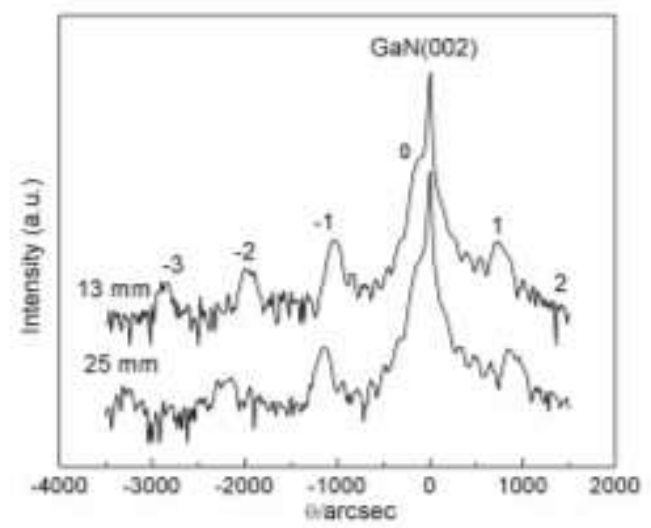

Figure 4. XRD $\omega-2 \theta$ scanning patterns of samples (0002) plane

Figure 4 shows the XRD $\omega-2 \theta$ scanning patterns of samples. The dominant peak is GaN (0002) diffraction peak, the left peak is the InGaN level 0 diffraction peak, the average In composition could be calculated from the space between $\mathrm{GaN}$ (0002) diffraction peak and InGaN level 0 diffraction peak [14]. Other peaks are the satellite peak, a cycle of MQW thickness could be calculated by the space of adjacent satellite [15]. And the advanced satellite peak number is responsible for the quality of MQW, the more satellite peak number, the better quality of MQW.

Table 1. The thickness of GaN barrier layer/InGaN well layer and In composition in InGaN well layer with different showerhead gap position

\begin{tabular}{|c|c|c|c|}
\hline Sample & Barrier (nm) & Well (nm) & $\begin{array}{c}\text { In } \\
\text { composition } \\
(\%)\end{array}$ \\
\hline $13 \mathrm{~mm}$ & 14.80 & 3.37 & 6.94 \\
\hline $25 \mathrm{~mm}$ & 12.38 & 3.29 & 5.38 \\
\hline
\end{tabular}

When increasing gap position, the pre-reaction gives rise to the transmission loss of reactants, which decreases the effective reactant reaching to the substrate, so the growth rate is reduced, quantum barrier layer, well layer and In components rapidly decreases; Compared with the well layer, the thickness of the barrier layer varies more sharply, this is because they adopt different Ga source for epitaxial growth. TMGa is adopted to grow barrier layer, and it is easy to dissociation, which can release more chemically active methyl radical, pre-reacted with $\mathrm{NH}_{3}$ violently, which give rise to consume more reactants, thus the thickness of barrier layer varies more dramatically. However, the epitaxial growth of well layer adopts TEGa, the hydrogen elimination is more likely to occur, to generate molecules containing metal - hydrogen bond and stable double olefin bonds, which is not easy to pre-react with $\mathrm{NH}_{3}$, reagent consumption is relatively small, thus the thickness of well layer is relatively stable with gap adjustment. From figure 4, we can see that with the increase of gap position, the intensity of satellite peak weakened and the FWHM broadens, the steep degree of MQW interface gradually becomes poor. However, this is contradicted with AFM results. This may be caused by the pre-reacted deposition in barrier layer.The pre-reacted deposition increases with the increasing gap position, which affects the growth of subsequent MQW, thus lead to a less steep interface. However, the surface becomes flat after a certain atomic layer thickness.

\section{Optical charactor}

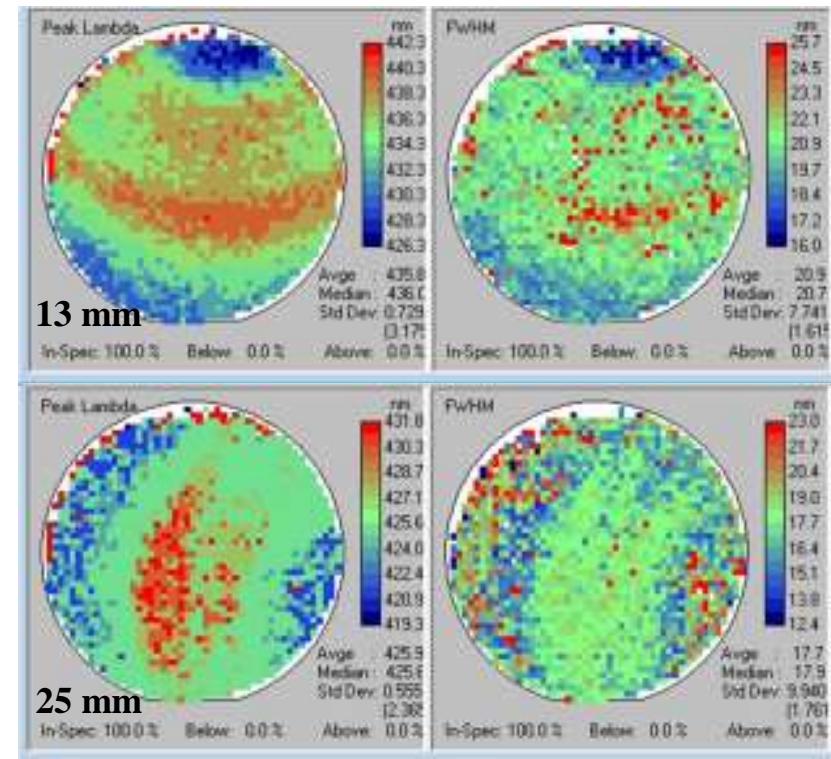

Figure 5. The PL mapping of emitting wavelength and FWHM of samples

The PL mapping of emitting wavelength and FWHM of samples are shown in figure 5. With the increase of gap position, the average emitting wavelength becomes blue shift, which is consistent with the XRD results. There may be two reasons: on one side, the blue shift is caused by the decrease of In component; on the other hand, the thinner thickness would weaken quantum confinement stark effect (QCSE).With the increase of gap position, the average FWHM of PL spectrum decreases, which indicated a uniform distribution of In component [16]. The reasons are following: on one hand, the increase gap position lead to the uniformity of sample; on the other hand, it is easier to distribute uniformly of In component in less In component sample, as result of decreasing FWHM.

\section{CONCLUSION}

In this paper, two InGaN/GaN MQW samples were studied at different gap position. With the increase of gap position, the surface roughness of MQW decrease, but interface steep deteriorated; also, the thickness of well layer, barrier layer and In component decreases. Compared with well layer, the thickness of barrier layer varied more significant. With the increase of gap position, the thickness of MQW is decreases, and the emitting wavelength of PL spectrum became blue shift; at the same time, In component distributes more uniformly.

\section{REFERENCES}

[1] E.F. Schubert. Light-emitting Diodes [M]. London: Cambridge University Press, 2006 
[2] J. Wu, W. Walukiewicz, K. M. Yu, et al. Unusual properties of the fundamental band gap of InN [J]. Applied physics letters, 2002, 80 (21):3967-3969

[3] C A Tran, A Osinski, R F Karlicek, et al. Growth of InGaN/GaN multiple-quantum-well blue light-emitting diodes on silicon by metalorganic vapor phase epitaxy [J]. Appl. Phys. Lett. 1999, 75 (11):1494-1496.

[4] G Y Zhang, Z J Yang, Y Z Tong, et al. InGaN/GaN MQW high brightness LED grown by MOCVD [J]. Optical Materials 2003, 23:183-186

[5] C L Mo, W Q Fang, Y Pu, et al. Growth and characterization of InGaN blue LED structure on $\mathrm{Si}(111)$ by MOCVD [J]. J. Cryst. Growth 2005, 285:312-317.

[6] Y. Yorozu, M. Hirano, K. Oka, and Y. Tagawa, "Electron spectroscopy studies on magneto-optical media and plastic substrate interface," IEEE Transl. J. Magn. Japan, vol. 2, pp. 740 741, August 1987 [Digests 9th Annual Conf. Magnetics Japan, p. 301, 1982].

[7] Z C Feng. III-Nitride Semiconductor Materials. London: Imperial College Press, 2006: 73.

[8] T G Mihopoulos, S G Hummel, K F J Jensen. Simulation of flow and growth phenomena in a close-spaced reactor [J]. J. Cryst. Growth 1998, 195:725-732
[9] X Y HU, H X Chen. Numerical-simulation-based design and verification of MOCVD reactor [J]. Equipment for Electronic Products Manufacturing 2008, 162:37-41.

[10] Z Ran, Z Hong, L X Lin. Numerical study of transport phenomena in a radial flow MOCVD reactor with three-separate vertical inlets [J]. Chinese Journal of Semiconductors 2005, 26 (5): 977-982.

[11] Y Chen, T Takeuchi, H Amano,et al. Pit formation in GaInN quantum wells[J].Appl.Phys. Lett.,1998,72(6):710-712

[12] H K Cho, J Y Lee, C S Kim, et al. Microstructural characterization of $\mathrm{InGaN} / \mathrm{GaN}$ multiple quantum wells with high indium composition[J].J.Cryst.Growth ,2001, 231:466-473

[13] S Mahanty, M Hao, T Sugahara, et al. V-shaped defects in InGaN/GaN multiquantum wells[J]. Materials Letters, 1999,41:6771

[14] S Srinivasan, R Liu, F Bertram, et al. A comparison of Rutherford Backscattering Spectroscopy and X-Ray Diffraction to determine the composition of thick InGaN epilayers [J]. Phys.Stat.Sol.(b), 2001, 228 (1):41-44

[15] M F Wu, S Q Zhou, S D Yao, et al. High precision determination of the elastic strain of InGaN/GaN multiple quantum wells [J]. J.Vac.Sci.Technol.B, 2004, 22 (3):920-924

[16] S J Chang, W C Lai, Y K Su, et al. InGaN/GaN multiquantum-well blue and green light-emitting diodes [J]. IEEE Journal on selected topics in quantum electronics, 2002, 8 (2):278-283 